\title{
A New Benzophenone from Lindera fruticosa
}

\author{
Myoung-Chong Song, Fikru Nigussie, ${ }^{+}$Hỵe-Joung Yang, and Nam-In Baek ${ }^{\circ}$ \\ Graduate School of Biotechnologv \& Plant Metabolism Research Center, Kvung Hee Universit, Suwon 4+6-701, Korea \\ "E-mail: nibaekakhuachr \\ Addis Ababa Cniversit, Faculty of Ieterinary Medicine. Debre Zeit, 34. Ethiopia \\ Received April 30, 2007
}

Key Words : Lindera fruticosa, 2.3-Dimethoxy-4-hydroxybenzophenone

Lindera fruticosa is a perennial shrub widely distributed in China. Nepal. India and Ethiopia. The root is a traditional anti-inflammatory medicine folk remedy but few studies on its active components has been reported. ' The potential therefore exists for new and valuable compounds to be discovered from L. friticosa. This paper describes the isolation and structural determination of a new benzophenone from the Li friticosa. Although this compound was reported by Kang et $a^{2}$ as a new compound from Sectridaca inappendiculata. the authors of this paper suggest that the identification of the compound carried out by Kang et $a f$. was incorrect.

\section{Experimental Section}

Instruments. HREIMS was recorded on a JEOL JMS 700 (JEOL. Tokyo. Japan). IR spectnum was run on a Perkin Elmer Spectrum One FT-IR spectrometer (Perkin Elmer. Norwalk. USA). ${ }^{1} \mathrm{H}-\mathrm{NMR}$ (400 MHz) and ${ }^{13} \mathrm{C}-\mathrm{NMR}(100$ $\mathrm{MHz}$ ) spectra were collected on a Varian Unity Inova AS 400 FT-NMR spectrometer (Varian. California. USA). ${ }^{3}$

Plant Materials. L. fruticosa roots were collected from a rural forest in Addis Ababa province. Ethiopia. by Prof. Fikru Nigussie and identified by Prof. Dae-Keun Kim. Woosuk University: Jeonju. Korea. A voucher specimen (KHU02031) was deposited in the Laboratory of Natural Products Chemistry. Kỵung Hee University. Suwon. Korea.

Isolation of 2,3-Dimethoxy-4-hydroxybenzophenone. The dried powdered roots $(1 \mathrm{~kg}$ ) were extracted with $80 \%$ aqueous methanol $(20 \mathrm{~L} \times 3)$ and concentrated in vacuo. The extracts were partitioned with $\mathrm{H}_{2} \mathrm{O}(2 \mathrm{~L})$ and $\mathrm{EtOAc}(2 \mathrm{~L} \times$ 3 ). The concentrated EtOAc fraction (LFE, 14 g) was subjected to silica gel column chromatography $(\mathrm{CC})(150 \mathrm{~g}$. $\Phi 5$ $\times 12 \mathrm{~cm})$ and eluted with a gradient of $\mathrm{CHCl}_{3}-\mathrm{MeOH}(10: 1$ $\rightarrow 7: 1$. v/v, $1 \mathrm{~L}$ of each). resulting in 12 fractions (LFEl LFE12). Fraction LFE3 [1.4 g. Ve/ Vt (elution volume/total volume) 0.10-0.15] was separated by RP- $18 \mathrm{CC}$ (150 g. $\Phi 4$ $\times 6 \mathrm{~cm})$ and eluted with $\mathrm{MeOH}-\mathrm{H}_{2} \mathrm{O}(1: 1 \rightarrow 2: 1.1 \mathrm{~L}$ of each $)$. resulting in 11 fractions (LFE3-1 LFE3-11). Fraction LFE34 (648 $\mathrm{mg}$. Ve/ $\mathrm{Vt} 0.13-0.22$ ) was subjected to silica gel CC (75 g. $\Phi 3.5 \times 9 \mathrm{~cm})$ and eluted with $n$-hexane-EtOAc $(2: 1$. v/s: $1.5 \mathrm{~L}$ ) yielding compound $1[230 \mathrm{mg}$. Ve/ $/ \mathrm{t} 0.13-0.26$ : TLC (Silica gel $60 \mathrm{~F}_{3 \leq 4}$ ) $\mathrm{R}_{f} 0.6, n$-hexane-EtOAc $\left.=1: 1\right]$.

2,3-Dimethoxy-4-hydroxybenzophenone (1): Colorless oil: IR $\left(\mathrm{CaF}_{2}\right.$ window in $\left.\mathrm{CHCl}_{2}\right) v_{\mathrm{max}} 3624,2924,1468$, 1225. $1065 \mathrm{~cm}^{-1}$ : EIMS $m=258[\mathrm{M}]^{-}$(100). 241 (100), 225 (30). 209 (58). 181 (100). $167(70) .151(16) .137(47) .105$ (74): HREIMS $m z 258.0865[\mathrm{M}]^{+}$(calcd for $\mathrm{C}_{15} \mathrm{H}_{3_{4}} \mathrm{O}_{4}=$ 258.0892): ${ }^{l} \mathrm{H}$ NMR (400 MHz. $\mathrm{CDCl}_{3}, \delta$ ) and ${ }^{13} \mathrm{C}$ NMR $\left(100 \mathrm{MHz}, \mathrm{CDCl}_{2}, \delta\right)$. see Table 1 .

\section{Results and Discussion}

The roots of $L$. fruticosa were extracted with $80 \%$ aqueous $\mathrm{MeOH}$, and the concentrated extract was partitioned with EtOAc and $\mathrm{H}_{2} \mathrm{O}$. From the EtOAc fraction. a benzophenone was isolated through repeated $\mathrm{SiO}_{2}$ and $\mathrm{ODS}$ column chromatography.

The compound was obtained as a colorless oil. The IR spectrum showed an absorption characteristic of phenolic alcohol $\left(3624 \mathrm{~cm}^{-1}\right)$, phenyl $\left(2924.1468 \mathrm{~cm}^{-1}\right)$ and ether (1225. $1065 \mathrm{~cm}^{-1}$ ). A molecular formula of $\mathrm{C}_{15} \mathrm{H}_{14} \mathrm{O}_{4}$ was determined by HREIMS ([M] ${ }^{+}, m z 258.0865$, calcd 258.0892 for $\left.\mathrm{C}_{15} \mathrm{H}_{14} \mathrm{O}_{4}\right)$. The ${ }^{1} \mathrm{H} N M R$ spectrum revealed an $\mathrm{AB}$ aromatic system at $\delta_{\mathrm{H}} 7.78(2 \mathrm{H}$, dd $J=8.4 .2 .0 \mathrm{~Hz}, \mathrm{H}-$ $\left.2^{\prime}, 6^{\prime}\right), \delta_{\mathrm{H}} 7.53\left(1 \mathrm{H}, \mathrm{dd}, J=8.4,2.0 \mathrm{~Hz}, \mathrm{H}-4^{\prime}\right)$. and $\delta_{\mathrm{H}} 7.41$ $\left(2 \mathrm{H}\right.$. dd. $\left.J=8.4,8.4 \mathrm{~Hz} . \mathrm{H}-3^{\prime}, 5^{\prime}\right)$ as a 1 -substituted benzene ring and $\delta_{\mathrm{H}} 7.06(1 \mathrm{H} . \mathrm{d}, J=8.4 \mathrm{~Hz} . \mathrm{H}-6)$ and $\delta_{\mathrm{H}} 6.74(1 \mathrm{H} . \mathrm{d}$. $J=8.4 \mathrm{~Hz}, \mathrm{H}-5)$ as a 1.2 .3 .4 -tetrasubstituted benzene ring with 2 methoxy at $\delta_{\mathrm{H}} 3.92\left(3 \mathrm{H}, \mathrm{H}-\mathrm{OCH}_{3}\right.$ at $\left.\mathrm{C}-3\right)$ and $\delta_{\mathrm{H}} 3.71$ $\left(3 \mathrm{H} . \mathrm{H}-\mathrm{OCH}_{3}\right.$ at $\left.\mathrm{C}-2\right)$. The ${ }^{13} \mathrm{C}$ NMR spectrum showed a characteristic non-chelated ketone carbon at $\delta_{\mathrm{C}} 195.2(\mathrm{C}-7)$. 5 quaternary sp' carbons at $\delta_{\mathrm{C}} 152.4(\mathrm{C}-4) . \delta_{\mathrm{C}} 151.9(\mathrm{C}-2)$. $\delta_{\mathrm{C}} 139.5(\mathrm{C}-3), \delta_{\mathrm{C}} 138.2\left(\mathrm{C}-1^{\prime}\right), \delta_{\mathrm{C}} 125.4(\mathrm{C}-1), 5$ methine $\mathrm{sp}^{2}$ carbons at $\delta_{\mathrm{C}} 132.6\left(\mathrm{C}-4^{\prime}\right), \delta_{\mathrm{C}} 125.8(\mathrm{C}-6), \delta_{\mathrm{C}} 110.1(\mathrm{C}-$ $5)$ including 2 overlapping signals at $\delta_{\mathrm{C}} 129.7\left(\mathrm{C}-2^{\prime}, 6^{\prime}\right), \delta_{\mathrm{C}}$ $128.1\left(\mathrm{C}-3^{\prime}, 5^{\prime}\right)$, and 2 methoxy signals at $\delta_{C} 61.5$ and $\delta_{C}$

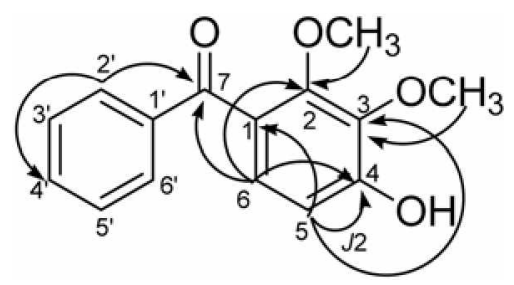

Figure 1. Chemical structure of 2,3-dimethoxy-4-hydroxyberzophenone from Lindera finticosa. The arrows indicate correlations between proton and carbon signals in the HMBC spectrum. 
Table 1. 'H (400 MHz, CDCl $)$ \& ${ }^{13} \mathrm{C}$ NMR $(100 \mathrm{MHz}, \mathrm{CDCl} 3)$ spectroscopic data of 2,3-dimethosy-4-hydroxy benzophenone from Lindera fruticosa and Securidaca inappendiculata ${ }^{2}$

\begin{tabular}{|c|c|c|c|c|}
\hline \multirow{2}{*}{$\begin{array}{l}\text { Carbon } \\
\text { Number }\end{array}$} & \multicolumn{2}{|c|}{ From Lindera finticosa } & \multicolumn{2}{|c|}{ From Securdaco inoppendiculata ${ }^{2}$} \\
\hline & $\delta_{\mathrm{H}}$ & $\dot{E}$ & $\delta_{\mathrm{H}}$ & $\delta_{C}$ \\
\hline 1 & & 125.4 & & 124.0 \\
\hline 2 & & 151.9 & & 152.5 \\
\hline 3 & & 139.5 & & 140.4 \\
\hline 4 & & 152.4 & & 154.4 \\
\hline 5 & $6.74(1 \mathrm{H}, \mathrm{d}, J=8.4 \mathrm{~Hz})$ & 110.1 & $6.72(1 \mathrm{H}, \mathrm{d}, j=8.4 \mathrm{~Hz})$ & 111.5 \\
\hline 6 & $7.06(1 \mathrm{H}, \mathrm{d}, J=8.4 \mathrm{~Hz})$ & 125.8 & $6.97(\mathrm{lH}, \mathrm{d}, J=8.4 \mathrm{~Hz})$ & 124.8 \\
\hline 7 & & 195.2 & & 194.7 \\
\hline$l^{\prime}$ & & 138.2 & & 138.2 \\
\hline $2^{\prime} / 6^{\prime}$ & $7.78(\mathrm{IH}, \mathrm{dd}, J=8.4,2.0 \mathrm{~Hz})$ & 129.7 & $7.67\left(\mathrm{IH}_{z} \mathrm{~d}_{s} J=8.0 \mathrm{~Hz}\right)$ & 129.7 \\
\hline $3^{1 / 5}$ & $7.41(\mathrm{IH}, \mathrm{dd}, J=8.4,8.4 \mathrm{~Hz})$ & 128.1 & $7.49(1 \mathrm{H}, \mathrm{t}, J=7.7 \mathrm{~Hz})$ & 128.2 \\
\hline 4 & $7.53(\mathrm{IH}, \mathrm{dd}, J=8.4,2.0 \mathrm{~Hz})$ & 132.6 & $7.61(1 \mathrm{H}, \mathrm{d}, J=7.5 \mathrm{~Hz})$ & 132.6 \\
\hline $\mathrm{OCH}_{3}(\mathrm{C} 2)$ & $3.71(3 \mathrm{H}, \mathrm{s})$ & 61.5 & $3.78(3 \mathrm{H}, \mathrm{s})$ & 61.1 \\
\hline $\mathrm{OCH}_{3}(\mathrm{C} 3)$ & $3.92(3 \mathrm{H}, \mathrm{s})$ & 61.1 & $360(3 \mathrm{H}, \mathrm{s})$ & 60.2 \\
\hline $\mathrm{OH}$ & - & - & $10.01(1 \mathrm{H}, \mathrm{s})$ & - \\
\hline
\end{tabular}

61.1. This spectroscopic data inplied that the compound was a trioxygenated benzophenone with a l-substituted benzene ring and a 1,2.3,4-tetrasubstituted benzene ring. In the gHMBC spectrun. every signal showed cross peaks by $\sqrt{3}$ correlation. An olefin methine signal at $\delta_{\mathrm{H}} 7.06(\mathrm{H}-6)$ showed cross peaks with a non-chelated ketone signal at $\delta_{C}$ $195.2(\mathrm{C}-7)$ and 2 olefun quaternary carbon signals at $\delta_{\mathrm{C}}$ $151.9(\mathrm{C}-2)$ and $\delta_{C} 152.4(\mathrm{C}-4)$. Another olefin methine signal at $\delta_{\mathrm{H}} 6.74(\mathrm{H}-5)$ showed cross peaks with 2 olefin quaternary carbon signals at $\delta_{C} 125.4(\mathrm{C}-1)$ and $\delta_{C} 139.5(\mathrm{C}$ 3). Two methoxy protons at $\delta_{\mathrm{H}} 3.92$ and $\delta_{\mathrm{H}} 3.71$ showed correlations with 2 olefin quaternary carbons at $\delta_{c} 139.5$ and $\delta_{C} 151.9$, respectively. The former correlation indicated 1 methoxy was at $\mathrm{C}-3$ and the latter correlation indicated another methosy was at $\mathrm{C}-2$ or $\mathrm{C}-4.42$ correlation was observed only between $\mathrm{H}-5$ and an olefin quatenary carbon at $\delta_{c} 152.4(\mathrm{C}-4)$, which showed no correlation with any methoxy proton. leading to the conclusion that another methoxy was at $\mathrm{C}-2$. Thus, the compound was identified as 2.3-dimethoxy-4-hydroxybenzophenone.

The 2,3-dimethoxy-4-hy'droxybenzophenone was reported to have been previously isolated from Sectridaca inappendiculata by Kang et $a l^{2}$ The ${ }^{1} \mathrm{H}$ - and ${ }^{13} \mathrm{C}-\mathrm{NMR}$ data suggested by Kang et al. showed many differences from the data proposed in this study (Table 1). The ${ }^{1} \mathrm{H}-\mathrm{NMR}$ data proposed by Kang et al. showed many the relative upfield shifts of $\mathrm{H}-5$ ( $-0.02 \mathrm{ppm})$. H-6 (-0.09 ppm). H-2/6' (-0.11 ppm) and C3-methosy $(-0.32 \mathrm{ppm})$, and the downfield shifts of $\mathrm{H}-3^{\prime} / 5^{\prime}(+0.08 \mathrm{ppm})$. H-1' (+0.08 ppm) and C2methoxy $(+0.07 \mathrm{ppm})$. The ${ }^{13} \mathrm{C} \cdot \mathrm{NMR}$ showed the variations mainly on the 1.2.3,4-tetrasubstituted benzene ring such as upfield shifts of $\mathrm{C}-\mathrm{l}(-1.4 \mathrm{ppm})$ and $\mathrm{C}-6(-1.0 \mathrm{ppm})$, and the downfield shifts of $\mathrm{C}-2(+0.6 \mathrm{ppm}) . \mathrm{C}-3(+0.9 \mathrm{ppm})$. C -4 $(+2.0 \mathrm{ppm})$. and $\mathrm{C}-5(+1.4 \mathrm{ppm})$. However, the proton signal of a hydrosy at $\delta_{\mathrm{H}} 10.01$ was observed, which indicates that there should be a $\mathrm{H}$-bond between a hydrogen of the hydroxy and an oxygen of a ketone (C-7) ${ }^{4 \cdot 6}$ Accordingly, the benzophenone isolated by Kang et al. should have a hydroxyl at C.2. indicating the chemical structure of the compound could be a 3.4-dimethoxy-2-hydrosybenzophenone. This evidence led to the conclusion that the 2,3-dimethoxy-4-hydrosybenzophenone isolated from $L$ finticosa was a new compound.

Acknowledgement. This study was supported by the SRC program of MOST/KOSEF (R11-2000-081) through the Plant Metabolism Research Center and Research Year Program (2006), Kyung Hee University, Korea.

\section{References}

1. Song. M. C.: Nigussie. F.: Jeong. T. S.: Lee. C. Y.: Regassa. F.: Markos. T.: Baek. N. I. J. Nat. Prod 2006. 69. 853-855.

2. Kang. W. Y.: Wang. Z. M.: Li, Z. Q: Xu. X. J. Hetv Chim. Acta 2005. $88,2771-2776$.

3. Song. M. C.: Kim. D. H.; Hong, Y. H.: Kim. D. K.: Chung. I. S.; Kim. S. H.: Park. M. H.: Kwon. B. M.: Lee. Y. H.: Baek. N. I. Agric Chem. Biotechol 2003. 46.118-121.

4. Ho. J. C.: Chen1. C. M. Phwochentstry 2002. 61.405-408.

5. Aksnes. D. W.; Standnes. A.; Andemen, O. M. Magn. Res. Chem. 1996. 3t, 823-826.

6. Cho. M. Bull. Korean Chem. Soc. 2006. 27. 1940-1960. 